\title{
EFEITO DE HERBICIDAS SOBRE A BIOTA DE INVERTEBRADOS DO SOLO EM ÁREA DE RESTAURAÇÃO FLORESTAL
}

\author{
Rafael Nogueira Scoriza ${ }^{(1) *}$, Alessandro de Paula Silva ${ }^{(2)}$, Maria Elizabeth Fernandes \\ Correia $^{(3)}$, Paulo Sérgio dos Santos Leles ${ }^{(4)}$ e Alexander Silva de Resende ${ }^{(3)}$
}

(1) Universidade Federal Rural do Rio de Janeiro, Instituto de Agronomia, Departamento de Solos, Programa de Pós-graduação em Agronomia - Ciência do Solo, Seropédica, Rio de Janeiro, Brasil.

(2) Instituto Federal do Norte de Minas Gerais, Campus Salinas, Salinas, Minas Gerais, Brasil.

(3) Empresa Brasileira de Pesquisa Agropecuária, Embrapa Agrobiologia, Seropédica, Rio de Janeiro, Brasil.

(4) Universidade Federal Rural do Rio de Janeiro, Instituto de Florestas, Departamento de Silvicultura, Seropédica, Rio de Janeiro, Brasil.

* Autor correspondente.

E-mail: rafaelscoriza@gmail.com

\section{RESUMO}

O controle químico de plantas daninhas é um método eficaz utilizado em florestas plantadas e em áreas de restauração florestal, favorecendo o desenvolvimento das mudas florestais e a redução dos custos de intervenção. Entretanto, se essa estratégia não for aplicada de maneira correta, pode-se tornar um potencial causador de impactos negativos aos organismos do solo e aos processos ecológicos que esses participam. Avaliou-se o efeito do uso de herbicidas na biota do solo por meio da amostragem da comunidade da fauna edáfica e testes ecotoxicológicos de reprodução e sobrevivência de Folsomia candida e Enchytraeus crypticus, em área-alvo de restauração florestal recém-implantada. $O$ delineamento experimental adotado foi de blocos casualizados com quatro tratamentos e cinco repetições. Os tratamentos consistiram da aplicação dos herbicidas pós-emergentes mesotrione $\left(0,4 \mathrm{~L} \mathrm{ha}^{-1}\right)$, fluazifop-P-butyl $\left(1,0 \mathrm{~L} \mathrm{ha} \mathrm{h}^{-1}\right) \mathrm{e}$ nicosulfuron (1,5 $\mathrm{L} \mathrm{ha}^{-1}$ ), aplicados em área total nas parcelas, mais o controle (sem aplicação). Na área de estudo, foram realizadas duas aplicações dos herbicidas com intervalos de seis meses, onde as parcelas foram correspondentes aos tratamentos em ambas as aplicações. Em cada parcela, foram realizadas quatro coletas de solo, sendo a primeira (coleta 1) seis dias antes da segunda aplicação dos herbicidas na área. As demais, após um (coleta 2), oito (coleta 3) e 22 dias (coleta 4) dessa mesma aplicação. A comunidade da fauna do solo foi avaliada por meio do método de funis de Berlese modificado, utilizando-se uma amostra de solo por parcela. Em laboratório, os indivíduos foram contados e identificados em grandes grupos. Os ensaios ecotoxicológicos de sobrevivência e reprodução de $F$. candida e E. crypticus foram realizados a 
partir de uma amostra composta de solo por tratamento. Um dia após a aplicação do herbicida mesotrione, verificou-se diminuição da riqueza média nos índices Shannon Weaver e Pielou e na reprodução de $E$. crypticus. Concluiu-se que a dupla aplicação dos herbicidas fluazifop-Pbuthyl e nicosulfuron, nas respectivas doses de 1,0 e 1,5 $\mathrm{L} \mathrm{ha}^{-1}$, não promoveu impactos negativos para os invertebrados do solo. Os efeitos negativos apresentados pelo mesotrione, mesmo que temporários, remeteu a uma ponderação sobre o uso desse em áreas de restauração florestal.

Palavras-chave: mesotrione, mesofauna, ecotoxicologia.

\title{
ABSTRACT: HERBICIDE USE IN DEGRADED FOREST AREAS IN RESTORATION: EFFECTS ON SOIL INVERTEBRATE BIOTA
}

\begin{abstract}
Chemical control of weeds is an effective method used in planted forests and in forest restoration, which plays an important role in development of forest seedlings and reduces costs of intervention. However, when this strategy is not properly applied, it may have negative impacts on soil organisms and on the ecological processes in which they take part. The goal of this study was to evaluate the effects of herbicide use on soil biota through sampling of the soil fauna community and through ecotoxicological tests on the survival and reproduction of Folsomia candida and Enchytraeus crypticus in a target area of a recently-established forest restoration. A randomized block experimental design was adopted, with four treatments and five replications. Treatments consisted of applications of three post-emergence herbicides

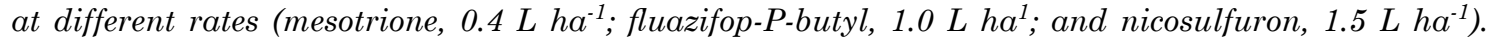
They were applied in the total area of the plots beyond an untreated check (without application). Two herbicide applications were carried out on the field under study at intervals of six months, and the plots corresponded to the treatments in both applications. A total of four soil samples were collected from each plot: sample 1, six days before the second herbicide application in the area; sample 2, one day after the application; sample 3, eight days after the application; and sample 4, 22 days after the application. The soil fauna community was evaluated through a modified Berlese funnel method using one soil sample per plot. Organisms were counted and identified in large groups in the laboratory. Ecotoxicological tests focusing on survival and reproduction of F. candida and E. crypticus were performed on one composite soil sample per treatment. One day after mesotrione application (sample 2), a strong decline was observed in average richness of organisms, Shannon's diversity index, and the Pielou uniformity index, as well as reproduction of E. crypticus. The double application of the herbicides fluazifop-P-butyl and nicosulfuron does not cause negative impacts on soil invertebrates, whereas the application of mesotrione has negative effects, even if temporarily. Great care must be taken in applying it in forest restoration.
\end{abstract}

Keywords: mesotrione, mesofauna, ecotoxicology.

\section{INTRODUÇÃO}

O sucesso de projetos para a restauração florestal está associado a diversos fatores, como a disponibilidade de nutrientes no solo, qualidade das sementes florestais, presença de herbivoria e competição com plantas daninhas (Holl et al., 2000). Entre esses, a competição das gramíneas com as mudas florestais por recursos-chave como água, luz e nutrientes (Victoria Filho, 1987; Machado et al., 2013) requer intervenções que eliminem a presença delas, facilitando ou mesmo propiciando o reestabelecimento da vegetação florestal (Elgar et al., 2014).

$\mathrm{O}$ uso de herbicidas em áreas florestais tem crescido nos últimos anos, principalmente pela comprovada eficiência deles no controle de plantas daninhas (Machado et al., 2013), resultando no aumento da sobrevivência das mudas arbóreas (Baer e Groninger, 2004), e pela diminuição dos custos de formação dos povoamentos arbóreos (Toledo et al., 1996). Nas plantações, seu uso pode auxiliar no preparo da área antes do plantio e após a colheita (Shepard et al., 2004); na restauração, é autorizado inclusive em unidades de conservação, em caráter emergencial (Brasil, 2012). Apesar das vantagens, o uso desses em áreas florestais geram expectativas negativas à sociedade sobre possíveis impactos ambientais. Essas advêm da grande porção dos pesticidas e seus resíduos que atingem o solo após a aplicação direta e, ou, foliar (Chaabane et al., 2008; Gill e Garg, 2014; Darine et al., 2015), com potencial de causar impactos negativos em organismos do solo, no meio ambiente e na saúde humana (Shepard et al., 2004). Além disso, há o risco de poluir ecossistemas aquáticos próximos, pela dispersão da pulverização, lixiviação no solo ou escorrimento superficial (Bonnet et al., 2008). 
Os compostos utilizados como pesticidas possuem grande abrangência de efeitos em toda a comunidade ecológica do solo (Mirsal, 2008); podem alterar o $\mathrm{C}$ microbiano do solo (Camelo et al., 2011), entrar na cadeia alimentar, bioacumular nos organismos e influenciar invertebrados do solo (Gill e Garg, 2014). Com isso, altera-se o funcionamento do solo (Cortet et al., 1999), como os processos de decomposição vegetal (Hendrix e Parmelee, 1985), a manutenção da estrutura do solo, a transformação e a mineralização da matéria orgânica e o controle de organismos considerados pragas (Gill e Garg, 2014). Há autores que concluem que há efeitos negativos do uso de herbicidas sobre a estruturação da comunidade de invertebrados do solo, como colêmbolos (Haque et al., 2011), nematoides (Zhao et al., 2013), minhocas (García-Pérez et al., 2014) e diversos grupos de microrganismos (Crouzet et al., 2010; Camelo et al., 2011; Karpouzas et al., 2014; Darine et al., 2015).

Para a restauração florestal, visando maior segurança, pesquisas devem ser realizadas para identificar efeitos ecológicos do manejo das gramíneas por herbicidas (Gerlach et al., 2013). As avaliações in situ são úteis por considerar a existência de grande número de espécies expostas a um ou mais estressores, o que aumenta a probabilidade de alguma espécie ser mais sensível que outra na detecção da contaminação (Rodrigues et al., 2011). Os bioensaios ex situ, de sobrevivência e reprodução, podem predizer a probabilidade de ocorrer efeitos adversos futuros (prospectivo) ou avaliar a probabilidade de efeitos serem causados pela exposição histórica (retrospectivo) (Usepa, 1998; Alves et al., 2014). Para esses ensaios, podem ser utilizados anelídeos do gênero Enchytraeus e collembola Folsomia candida. Ambos são ecologicamente relevantes por estarem presentes em altas densidades populacionais no solo de todo o mundo, apresentarem alta permeabilidade cutânea e participarem ativamente em processos de decomposição no solo (Fountain e Hopkin, 2005; $\mathrm{ABNT}, 2012$ ).
Considerando que o uso de defensivos na restauração florestal não deva causar efeitos em organismos não-alvo da biota do solo, avaliou-se o efeito do uso de três herbicidas pós-emergentes, de diferentes mecanismos de ação, por meio da avaliação in situ da comunidade da fauna edáfica e da ex situ da sobrevivência e reprodução de Folsomia candida e Enchytraeus crypticus.

\section{MATERIAL E MÉTODOS}

\section{Histórico da área}

O estudo foi conduzido em uma área localizada no campo experimental da Embrapa Agrobiologia, Seropédica, Rio de Janeiro, onde está sendo estudada a toxicidade de três herbicidas em espécies florestais nativas. A área experimental possui as seguintes características granulométrica e química do solo na profundidade de $0,20 \mathrm{~m}$ : textura franco-arenosa (argila 166; silte 125; areia $\left.709 \mathrm{~g} \mathrm{~kg}^{-1}\right) ; \mathrm{pH}\left(\mathrm{H}_{2} \mathrm{O}\right) 5,0 ; \mathrm{Al}^{3+} 0,2 \mathrm{cmol}_{\mathrm{c}} \mathrm{dm}^{-3}$; $\mathrm{H}+\mathrm{Al} 4,0 \mathrm{cmol}_{\mathrm{c}} \mathrm{dm}^{-3} ; \mathrm{Ca}^{2+} 0,9 \mathrm{cmol}_{\mathrm{c}} \mathrm{dm}^{-3}$; $\mathrm{Mg}^{2+}$ 0,7 $\mathrm{cmol}_{\mathrm{c}} \mathrm{dm}^{-3} ; \mathrm{K} 49,3 \mathrm{mg} \mathrm{L}{ }^{-1} ; \mathrm{P} \mathrm{1,3} \mathrm{mg} \mathrm{L}^{-1}$; e carbono orgânico $0,9 \mathrm{dag} \mathrm{kg}^{-1}$.

$\mathrm{Na}$ área experimental, onde predomina o capim braquiária (Urochloa humidicola (Rendle) Schweick), fez-se o plantio de quatro espécies florestais nativas: Schinus terebinthifolius Raddi, Gochnatia polymorpha (Less.) Cabrera, Inga laurina (Sw.) Willd e Ceiba speciosa (A.St.-Hil.) Ravenna, em março de 2014.

Os tratamentos consistiram da aplicação de três herbicidas (em área total) e o controle (sem aplicação). Os herbicidas aplicados foram o mesotrione (Callisto ${ }^{\circledR} ; 0,4 \mathrm{~L} \mathrm{ha}^{-1}$ p.c.), fluazifop-P-butyl (Fusilade ${ }^{\circledR} ; 1,0$ L ha $^{-1}$ p.c.) e nicosulfuron (Sanson ${ }^{\circledR}$; $1,5 \mathrm{~L} \mathrm{ha}^{-1}$ p.c.), adotando-se as doses recomendadas para o controle de gramíneas (Quadro 1). Efetuou-se

Quadro 1. Principais características dos herbicidas aplicados na área experimental de restauração florestal

\begin{tabular}{|c|c|c|c|}
\hline Característica & Mesotrione & Fluazifop-P-buthyl & Nicosulfuron \\
\hline Comumente utilizado em cultura & Milho & Dicotiledôneas: soja & Milho \\
\hline Modo de ação na planta & $\begin{array}{c}\text { Causa degradação } \\
\text { oxidativa da clorofila e da } \\
\text { membrana plasmática }\end{array}$ & $\begin{array}{l}\text { Bloqueio da produção de } \\
\text { fosfolipídios usados em } \\
\text { membranas celulares }\end{array}$ & $\begin{array}{l}\text { Interrupção da síntese } \\
\text { proteica e DNA }\end{array}$ \\
\hline Tempo de meia vida no campo (dia) & 5,0 - Não persistente & 8,2 - Não persistente & 19,3 - Não persistente \\
\hline Solubilidade em água a $20^{\circ} \mathrm{C}\left(\mathrm{mg} \mathrm{L}^{-1}\right)$ & 160 - Moderada & 0,93 - Baixa & 7.500 - Alta \\
\hline Adsorção ao solo e mobilidade $\left(\mathrm{K}_{\mathrm{oc}}\right)$ & $\begin{array}{c}122 \text { - Moderadamente } \\
\text { móvel }\end{array}$ & 3394 - Levemente móvel & 30 - Móvel \\
\hline Pressão de vapor a $25^{\circ} \mathrm{C}(\mathrm{MPa})$ & $5,7 \times 10^{-3}$ - Não volátil & 0,12 - Não volátil & $8,0 \times 10^{-7}$ - Não volátil \\
\hline $\begin{array}{l}\text { Coeficiente de distribuição entre } \\
\text { octanol-água em pH } 7 \text { a } 20^{\circ} \mathrm{C}(\log \mathrm{P})\end{array}$ & 0,11 - Baixo & 4,5 - Alta & 0,61 - Baixo \\
\hline
\end{tabular}


a aplicação dos herbicidas nas mesmas parcelas em ambas as aplicações, utilizando-se a mesma dose e tecnologia de aplicação.

O delineamento experimental foi em blocos casualizados, com quatro tratamentos e cinco repetições, onde as parcelas compreenderam uma área útil de $41,6 \mathrm{~m}^{2}$. Foram realizadas duas aplicações, sendo a primeira em março de 2014 e a segunda em outubro de 2014, utilizando-se um pulverizador costal manual, com válvula reguladora de pressão acoplada a uma barra provida de bico com ponta de pulverização do tipo TT 11002, aplicando um volume de calda herbicida correspondente a $300 \mathrm{~L} \mathrm{ha}^{-1}$.

\section{Coleta do solo e da fauna edáfica para os ensaios ecotoxicológicos}

A coleta de solo para os ensaios ecotoxicológicos e a amostragem da fauna edáfica foram realizadas em quatro momentos. A primeira (coleta 1) foi feita aos seis dias antes da segunda aplicação dos herbicidas; e, as demais, após um (coleta 2), oito (coleta 3) e 22 dias (coleta 4) após a aplicação. Essa estratégia considerou a possibilidade de efeitos em longo prazo da primeira aplicação (coleta 1) e o monitoramento dos efeitos em curto prazo, após a segunda aplicação (coletas 2, 3 e 4). Os intervalos foram definidos como iguais ou múltiplos de sete dias, com base no tempo necessário para extrair os organismos da fauna edáfica no funil de Berlese e no tempo de meia-vida dos herbicidas (Quadro 1).

A amostragem da fauna edáfica baseou-se no método do funil de Berlese. Para isso, coletou-se uma amostra de solo por parcela, utilizando uma sonda metálica cilíndrica com $0,08 \mathrm{~m}$ de diâmetro, sendo inserida no solo até $0,05 \mathrm{~m}$ de profundidade $\left(251,2 \mathrm{~cm}^{3}\right.$ de solo). Como a aplicação do herbicida é superficial e dirigida à gramínea, a matéria vegetal (viva e morta) acima do solo coletado foi considerada na amostragem. Em laboratório, cada amostra foi inserida em um cilindro metálico com uma grade de $2 \mathrm{~mm}$ em sua base, submetido a uma lâmpada incandescente de $20 \mathrm{~W}$ por sete dias, com o propósito de os organismos reagirem ao calor e moverem-se para baixo, caindo no frasco coletor contendo formol a $1 \%$. Adicionalmente, uma pequena amostra com estrutura preservada de cada parcela $(\approx 10 \mathrm{~g})$ foi pesada antes e após ser submetida à estufa a $105^{\circ} \mathrm{C}$ por $24 \mathrm{~h}$, possibilitando a avaliação da umidade percentual do solo.

Para os ensaios ecotoxicológicos, coletaram-se amostras de solo na profundidade de 0,00-0,05 m, com o auxílio de uma enxada, em dois pontos aleatórios de cada parcela, formando uma amostra composta. No mesmo dia, em laboratório, cada amostra foi peneirada em malha de $2 \mathrm{~mm}$ e pesados $100 \mathrm{~g}$, formando, para cada tratamento, uma amostra composta, representativa da área experimental. Essas foram submetidas a dois ciclos intercalados de congelamento e descongelamento de $24 \mathrm{~h}$ cada, visando a completa eliminação da fauna edáfica (Pesaro et al., 2003).

\section{Ensaios ecotoxicológicos e triagem da fauna edáfica}

Os indivíduos coletados da fauna edáfica foram contados e identificados em grandes grupos taxonômicos, de acordo com as descrições fornecidas por Dindal (1990). Sequencialmente, calcularam-se o número de indivíduos, a riqueza total, a riqueza média e os índices de diversidade de Shannon Heaver $\left(\mathrm{H}=-\sum\right.$ pi log pi) e a equabilidade de Pielou $\left(\mathrm{e}=\mathrm{H} \log \mathrm{R}^{-1}\right)$, em que pi é a frequência relativa de indivíduos de cada grupo taxonômico; e $\mathrm{R}$, a riqueza definida como o número de diferentes unidades taxonômicas coletadas em cada área avaliada (Odum e Barrett, 2011). A ausência ou presença de grupos também foi considerada por sua simples ocorrência.

Os ensaios com $F$. candida e E. crypticus seguiram as normas obrigatórias e recomendadas da NBR ISO 11267 (ABNT, 2011) e NBR ISO 16387 (ABNT, 2012), respectivamente. Para ambos, o solo foi previamente umedecido com água destilada entre 40 a $60 \%$ da sua capacidade de retenção de água. Os ensaios foram conduzidos em câmara de incubação com controle de temperatura (18 a $22{ }^{\circ} \mathrm{C}$ ), fotoperíodo ( $16 \mathrm{~h}$ de luz para $8 \mathrm{~h}$ de escuro) e intensidade luminosa (400 a 800 lux). A umidade do solo foi controlada semanalmente pelo peso inicial dos recipientes-testes, adicionando água destilada quando necessário. Os organismos também foram submetidos ao substrato padrão "solo artificial tropical" (ABNT, 2014), com cinco repetições cada, para avaliar a adequação às condições ambientais dos ensaios. A umidade do solo foi mantida constante ao longo do experimento, e a variação do $\mathrm{pH}$ entre o início e o final foi igual ou menor que 0,7.

Os indivíduos de E. crypticus foram criados em placas de petri contendo meio ágar, em um ambiente de temperatura constante de $20 \pm 2{ }^{\circ} \mathrm{C}$. Utilizaram-se recipientes cilíndricos transparentes $(40 \mathrm{~mL})$ com tampa, contendo $20 \mathrm{~g}$ do equivalente do solo natural seco e $50 \mathrm{mg}$ de aveia em flocos finos (alimento), em cinco replicatas. Em cada recipiente, foram inseridos 10 indivíduos ovados, selecionados e coletados em estereomicroscópio. Uma sexta réplica, sem organismos e alimento, foi utilizada para avaliar o $\mathrm{pH}$ e a umidade ao final. Semanalmente, foram adicionados superficialmente $25 \mathrm{mg}$ de aveia nos recipientes com organismos. $\mathrm{O}$ ensaio teve duração de 21 dias, visando evitar um número excessivo de juvenis (Niva et al., 2010). Ao final, os recipientes foram preenchidos com solução de Rosa de Bengala a $1 \%$ de etanol, promovendo a coloração dos organismos e facilitando a contagem dos indivíduos adultos e juvenis sob estereomicroscópio.

Os indivíduos de $F$. candida foram criados em substrato formado por gesso e carvão ativado, na proporção de 8:1, em um ambiente de temperatura 
constante de $20 \pm 2{ }^{\circ} \mathrm{C}$. No ensaio, utilizaram-se recipientes cilíndricos transparentes $(80 \mathrm{~mL})$, contendo $30 \mathrm{~g}$ de solo natural úmido e $2 \mathrm{mg}$ de fermento seco granulado (alimento), em cinco réplicas. Em cada recipiente, foram adicionados 10 indivíduos sincronizados com 10 a 12 dias de vida. Uma sexta réplica, sem organismos e alimento, foi utilizada para avaliar o $\mathrm{pH}$ e a umidade ao final. Após 14 dias, foram adicionados superficialmente $2 \mathrm{mg}$ de fermento granulado nos recipientes com organismos. $\mathrm{O}$ ensaio teve duração de 28 dias. Ao final, o solo contendo os organismos foi colocado em um recipiente maior $(500 \mathrm{~mL})$, sendo adicionada água destilada até sua total cobertura e promovendo leve agitação com espátula. Esse procedimento causa a flutuação dos organismos vivos. Algumas gotas de tinta de carimbo na cor azul-escura foram utilizadas para destacar os organismos. Os indivíduos adultos foram contados visualmente; e os juvenis, pela contagem manual em fotografias no software Image Toll ${ }^{\circledR}$.

\section{Análise dos dados}

Os dados foram testados quanto à homogeneidade das variâncias dos tratamentos e normalidade em nível de $95 \%$ de probabilidade, e constatou-se não haver necessidade de transformação. Os tratamentos dos herbicidas foram comparados apenas com o controle (solo natural) pelo teste Dunnet $(\alpha=0,05)$, realizado no software Action ${ }^{\circledR}$.

\section{RESULTADOS E DISCUSSÃO}

Os herbicidas pós-emergentes aplicados na área experimental de restauração florestal não promoveram relevantes modificações na comunidade da mesofauna do solo. Apenas o mesotrione causou modificações significativas na riqueza média de grupos, sendo essa detectada apenas um dia após sua aplicação, não refletindo na riqueza total. Nessa avaliação, tanto os índices Shannon Weaver e Pielou do tratamento com aplicação do mesotrione foram menores que o controle (Quadro 2). Ressalta-se que a influência da sazonalidade das condições ambientais ocorridas desde a implantação não foi considerada neste estudo, uma vez que em todos os períodos de coleta nas parcelas, onde foram aplicados os herbicidas, foram realizadas também coletas nas parcelas que dispunham do tratamento-controle (sem aplicação).

O índice de Pielou variou entre 0,35 e 0,59, o que demonstrou dominância na comunidade em todas as coletas, mesmo onde não houve a aplicação de herbicidas. O grupo Acari foi o responsável por essa dominância, representando de 40 a $76 \%$ do número de indivíduos na comunidade composta

Quadro 2. Caracterização da comunidade da mesofauna do solo na área experimental de restauração florestal submetida à aplicação de herbicidas

\begin{tabular}{|c|c|c|c|c|c|}
\hline Herbicida & Indivíduos & Riqueza total & Riqueza média & Shannon & Pielou \\
\hline & \multicolumn{5}{|c|}{ Coleta 1 (6 dias antes da $2^{\mathrm{a}}$ aplicação dos herbicidas) } \\
\hline Mesotrione & $104 \pm 16$ & 14 & 8,2 & 2,21 & 0,58 \\
\hline Fluazifop-P-buthyl & $205 \pm 79$ & 13 & 8,2 & 1,81 & 0,49 \\
\hline Nicosulfuron & $176 \pm 45$ & 15 & 8,8 & 2,26 & 0,58 \\
\hline \multirow[t]{2}{*}{ Controle } & $193 \pm 47$ & 20 & 11,8 & 2,46 & 0,57 \\
\hline & \multicolumn{5}{|c|}{ Coleta 2 ( 1 dia após a $2^{\mathrm{a}}$ aplicação dos herbicidas) } \\
\hline Mesotrione & $75 \pm 23$ & 13 & $5,4^{*}$ & 1,31 & 0,35 \\
\hline Fluazifop-P-buthyl & $116 \pm 22$ & 17 & 8,4 & 1,79 & 0,44 \\
\hline Nicosulfuron & $72 \pm 22$ & 13 & 6,2 & 1,45 & 0,39 \\
\hline \multirow[t]{2}{*}{ Controle } & $112 \pm 29$ & 14 & 8,2 & 1,55 & 0,41 \\
\hline & \multicolumn{5}{|c|}{ Coleta 3 (8 dias após a $2^{\text {a }}$ aplicação dos herbicidas) } \\
\hline Mesotrione & $37 \pm 12$ & 11 & 5,4 & 1,80 & 0,52 \\
\hline Fluazifop-P-buthyl & $54 \pm 17$ & 10 & 5,4 & 1,94 & 0,59 \\
\hline Nicosulfuron & $75 \pm 28$ & 12 & 6,0 & 1,98 & 0,55 \\
\hline \multirow[t]{2}{*}{ Controle } & $79 \pm 36$ & 14 & 6,2 & 1,92 & 0,50 \\
\hline & \multicolumn{5}{|c|}{ Coleta 4 (22 dias após a $2^{a}$ aplicação dos herbicidas) } \\
\hline Mesotrione & $246 \pm 36$ & 17 & 7,8 & 1,62 & 0,40 \\
\hline Fluazifop-P-buthyl & $248 \pm 71$ & 13 & 7,0 & 1,99 & 0,54 \\
\hline Nicosulfuron & $349 \pm 127$ & 21 & 9,8 & 1,58 & 0,36 \\
\hline Controle & $335 \pm 92$ & 19 & 7,8 & 1,57 & 0,37 \\
\hline
\end{tabular}

*Média inferior ao controle, pelo teste Dunnet $(\alpha=0,05)$. 
Mesotrione
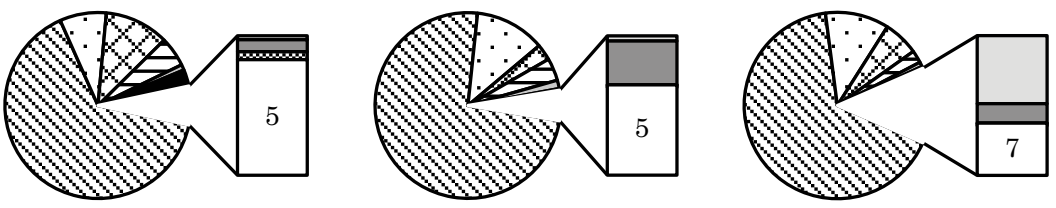

Coleta 2
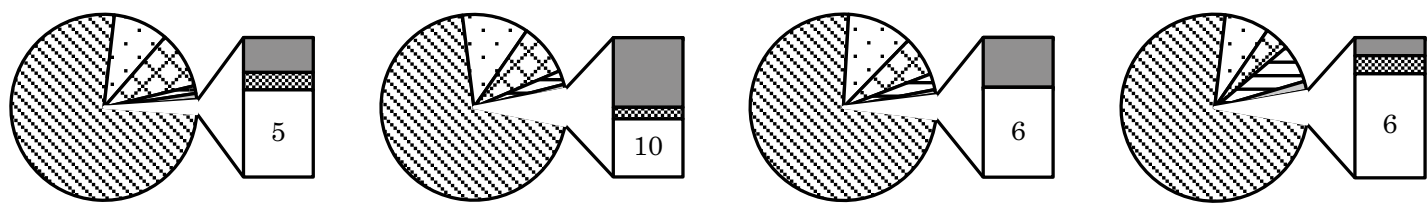

Coleta 3
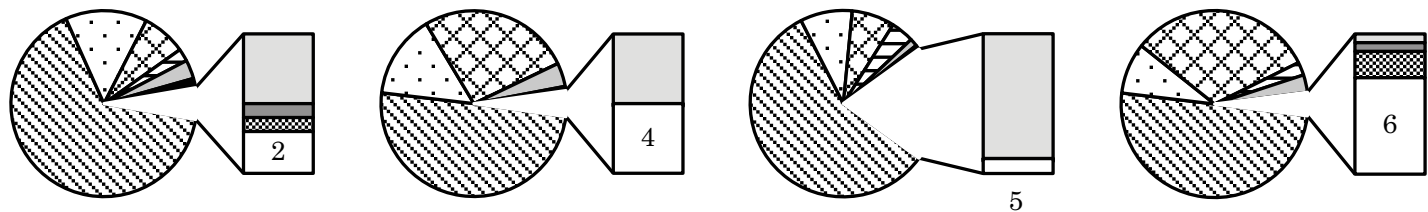

Coleta 4
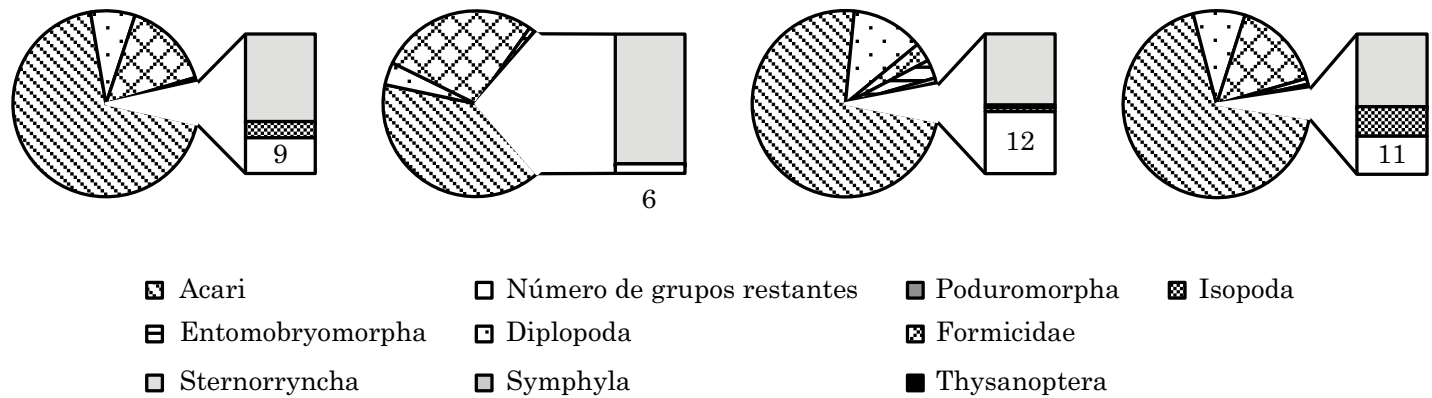

$$
\begin{aligned}
& \text { Número de grupos restantes } \\
& \text { Diplopoda } \\
& \square \text { Symphyla }
\end{aligned}
$$

$$
\begin{aligned}
& \text { Poduromorpha } \\
& \text { a Formicidae } \\
& \text { - Thysanoptera }
\end{aligned}
$$

Figura 1. Distribuição dos principais grupos da comunidade da fauna do solo em área experimental de restauração florestal submetida à aplicação de herbicidas pós-emergentes. Coleta 1 realizada seis dias antes da $2^{\mathrm{a}}$ aplicação dos herbicidas. Coletas 2,3 e 4 realizadas um, oito e 22 dias, após a $2^{\mathrm{a}}$ aplicação dos herbicidas, respectivamente.

por um total de 27 grupos. Os grupos Diplopoda e Formicidae também tiveram relevância numérica na comunidade (Figura 1). Para esses e para os demais grupos, não houve diferenças entre os tratamentos com herbicidas em relação ao controle.

O controle das gramíneas exercido pelos herbicidas ou de forma manual (controle) pode ter causado a dominância dos grupos Acari, Diplopoda e Entomobryomorpha, uma vez que as funções exercidas por esses no solo são direta e indiretamente relacionadas com a decomposição de matéria vegetal, bem como com a ciclagem de nutrientes (Odum e Barrett, 2011; Menta, 2012). Os três grupos são comumente utilizados como indicadores para avaliar impactos ambientais (Fountain e Hopkin, 2005; Gerlach et al., 2013).

Considerando a ausência/presença de grupos na avaliação do uso dos herbicidas, não se verificou um padrão de relação. Destacou-se apenas casuais ausências de alguns grupos onde houve a aplicação de herbicida (Figura 1), como de Isopoda nas três primeiras coletas, onde 
foram aplicados nicosulfuron, e de Thysanoptera e Entomobryomorpha nas coletas 2 e 3, para a aplicação de fluazifop-P-buthyl. Para os grupos de menor representatividade numérica, Auchenorryncha esteve presente apenas nos locais onde houve a aplicação de herbicidas, entretanto, somente nas coletas 2 e 4 , o que pode indicar vantagem competitiva pela aplicação desses produtos químicos (Menta, 2012). O aumento de microartrópodes herbívoros pode até ser esperado em resposta à aplicação de herbicidas, pois são fontes de carbono disponíveis (Hendrix e Parmelee, 1985).

Para os ensaios ecotoxicológicos, a sobrevivência de $F$. candida e $E$. crypticus não foram reduzidas pelo uso dos herbicidas (Figura 2). No que tange à reprodução, verificou-se redução dessa de E. crypticus com a aplicação do herbicida mesotrione, porém apenas com o solo proveniente da coleta 2 .

Os critérios de validação de ambos os ensaios, em relação ao solo artificial e natural controle, foram atendidos e, portanto, as condições ambientais de temperatura e luminosidade não influenciaram nesses. Para F. cândida, a letalidade dos adultos não excedeu $20 \%$, a reprodução atingiu mais de 100 juvenis por recipiente e o coeficiente de variação da reprodução não excedeu $30 \%$. Para
$E$. crypticus, a letalidade dos adultos não excedeu $20 \%$, a reprodução atingiu mais de 50 juvenis por recipiente e o coeficiente de variação da reprodução não excedeu $50 \%$.

Poucos ensaios ecotoxicológicos são encontrados na literatura avaliando o efeito de herbicidas aplicados em solos naturais. Neste estudo, com exceção da inibição da reprodução de $E$. crypticus no solo da coleta 2 com mesotrione, os herbicidas não apresentaram efeitos na sobrevivência e reprodução dos organismos utilizados. Os enquitreídeos são comuns em muitos solos, principalmente nos primeiros $0,05 \mathrm{~m}$, e desempenham a função de decomposição (Cortet et al., 1999); o hábito deles de ingestão direta de solo pode ter aumentado sua exposição (ABNT, 2012). Ensaios com minhocas constataram aumento da possibilidade de alta ingestão do herbicida glifosato, em razão da alta adsorção desse com a argila do solo (García-Pérez et al., 2014). Estudando Eisenia fetida, em contato com papel-filtro, Wang et al. (2012) caracterizaram os herbicidas fluazifop-P-butyl e mesotrione como moderadamente tóxicos.

O efeito do herbicida mesotrione sobre a reprodução de E. crypticus apenas um dia após sua aplicação pode estar relacionado principalmente à dose, o que pode ter propiciado sua rápida dissipação no solo (Crouzet et al.,
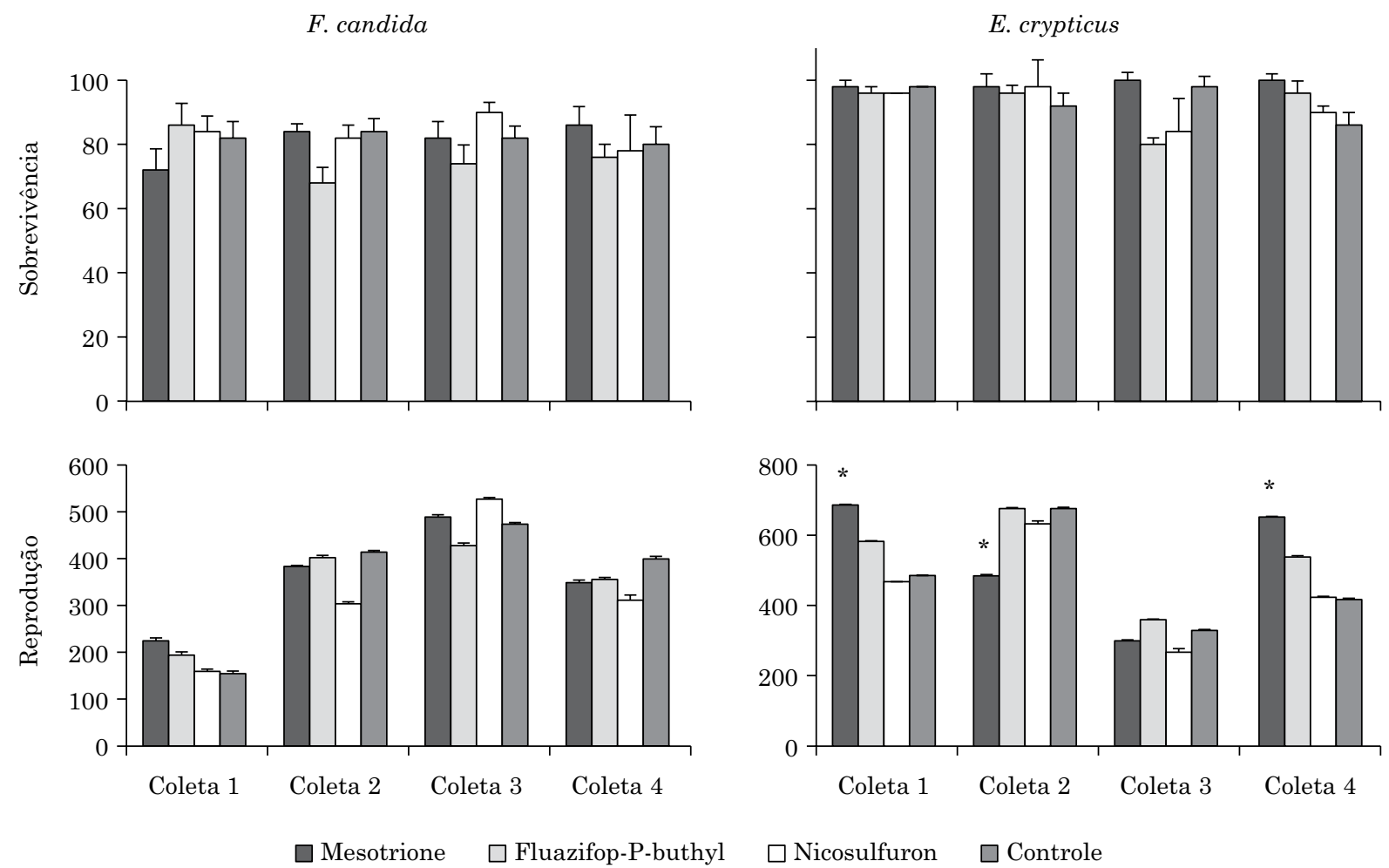

Figura 2. Sobrevivência e reprodução de F. candida e E. crypticus em solo proveniente de área experimental de restauração florestal submetido à aplicação de três herbicidas pós-emergentes. Coleta 1 realizada seis dias antes da $2^{\mathrm{a}}$ aplicação dos herbicidas. Coletas 2,3 e 4 realizadas um, oito e 22 dias, após a $2^{\mathrm{a}}$ aplicação dos herbicidas, respectivamente. * Média difere do controle, pelo teste Dunnet $(\alpha=0,05)$. 
2010), já que possui características de não persistência (Quadro 1). A rápida absorção pelas espécies daninhas (Mitchell et al., 2001) e a capacidade de degradação pelos microrganismos do solo (Batisson et al., 2009) são fatores que podem ser considerados.

Mesmo com efeito temporário, ainda permanece a questão: considerando os herbicidas analisados, por que apenas o mesotrione demonstrou algum efeito negativo na biota do solo? O mesotrione foi desenvolvido de uma fitotoxina natural para minimizar os impactos ambientais (Crouzet et al., 2010). As características dele (Quadro 1) não auxiliam na resposta dessa pergunta, pois apresenta menor solubilidade em água que o nicosulfuron e menor adsorção ao solo e mobilidade que o fluazifop-P-buthyl. Entretanto, sabe-se que esse herbicida atende a todos os critérios propostos pela United States Environmental Protection Agency (Usepa) para classificar o composto como contaminante para águas subterrâneas (Martinazzo et al., 2011).

Outra fonte de resposta pode ser a formulação do produto comercial Callisto ${ }^{\circledR}$, que é conhecidamente mais tóxico que o componente analítico mesotrione, por causa da presença de surfactantes, designado para aumentar a sua eficiência (Bonnet et al., 2008). Além disso, conhece-se pouco a respeito da ação dos metabólitos gerados pela degradação do mesotrione (Batisson et al., 2009) como o 4-methylsulfonyl-2-nitrobenzoic acid e o 2-amino-4-methylsulfonylbenzoic acid (AERU, 2015).

\section{CONCLUSÕES}

A utilização dos herbicidas fluazifop-P-buthyl e nicosulfuron para o controle de gramíneas influenciou a biota de invertebrados do solo, quando aplicados duas vezes na área e nas doses de 1,0 e 1,5 $\mathrm{L} \mathrm{ha}^{-1}$, respectivamente.

$\mathrm{O}$ herbicida mesotrione evidenciou-se com potencial de reduzir a riqueza de grupos da mesofauna e a reprodução de E. crypticus. Mesmo com efeitos temporários, o uso desse herbicida deve ser evitado em áreas de restauração florestal.

\section{REFERÊNCIAS}

Agriculture and Environment Research Unit - AERU. Pesticide properties DataBase. [Acessado em 6 fev 2015]. Disponível em: http://sitem.herts.ac.uk/aeru/ppdb.

Alves PRL, Cardoso EJBN, Martines AM, Sousa JP, Pasini A. Seed dressing pesticide on springtails in two ecotoxicological laboratory tests. Ecotoxicol Environ Safe. 2014;105:65-71.
Associação Brasileira de Normas Técnicas - ABNT. NBR 15537. Ecotoxicologia terrestre - Toxicidade aguda - Método de ensaio com minhocas (Lumbricidae). Rio de Janeiro: 2014.

Associação Brasileira de Normas Técnicas - ABNT. NBR ISO 11267. Qualidade do solo - Inibição da reprodução de Collembola (Folsomia candida) por poluentes do solo. Rio de Janeiro: 2011.

Associação Brasileira de Normas Técnicas - ABNT. NBR ISO 16387. Qualidade do solo - Efeitos de poluentes em Enchytraeidae (Enchytraeus sp.) - Determinação de efeitos sobre a reprodução e sobrevivência. Rio de Janeiro: 2012.

Baer SG, Groninger JW. Herbicide and tillage effects on volunteer vegetation composition and diversity during reforestation. Restor Ecol. 2004;12:258-67.

Batisson I, Crouzet O, Besse-Hoggan P, Sancelme M, Mangot $\mathrm{J}$, Mallet C, Bohatier J. Isolation and characterization of mesotrione-degrading Bacillus sp. from soil. Environ Pollut. 2009;157:1195-201.

Bonnet JL, Bonnemoy F, Dusser M, Bohatier J. Toxicity assessment of the herbicides sulcotrione and mesotrione toward two reference environmental microorganisms: Tetrahymena pyriformis and Vibrio fischeri. Arch Environ Contam Toxicol. 2008;55:576-83.

Brasil. Instrução normativa Ibama ${ }^{\circ} 7$, de 2 de jul. 2012. Diário Oficial da União [internet]. Brasília, DF: Instituto Brasileiro do Meio Ambiente; 2012. [Acessado em 26 jan. 2015]. Disponível em: http://www.institutohorus.org.br/download/marcos_legais/ INSTRUCAO_NORMATIVA_7_IBAMA_DE_02-07-2012 Registro_herbicidas.pdf.

Camelo GN, Santos JB, Lazari TM, Oliveira TA, Santos EA, Ferreira EA, Pereira GAM. Efeito do sistema de plantio e doses do nicosulfuron sobre a atividade microbiana do solo. Planta Daninha. 2011;29:829-35.

Chaabane H, Vulliet E, Calvayrac C, Coste C, Cooper J. Behaviour of sulcotrione and mesotrione in two soils. Pest Manage Sci. 2008;64:86-93.

Cortet J, Vauflery A, Poinsot-Balaguer N, Gomot L, Texier C, Cluzeau D. The use of invertebrate soil fauna in monitoring pollutant effects. Eur J Soil Biol. 1999;35:115-34.

Crouzet O, Batisson I, Besse-Hoggan P, Bonnemoy F, Bardot C, Poly F, Bohatier J, Mallet C. Response of soil microbial communities to the herbicide mesotrione: A dose-effect microcosm approach. Soil Biol Biochem. 2010;42:193-202.

Darine T, Alaeddine C, Fethi B, Ridha M. Fluazifop-P-butyl (herbicide) affects richness and structure of soil bacterial communities. Soil Biol Biochem. 2015;81:89-97.

Dindal DL. Soil biology guide. New York: Wiley; 1990.

Elgar AT, Freebody K, Pohlman CL, Shoo LP, Catterall CP. Overcoming barriers to seedling regeneration during forest restoration on tropical pasture land and the potential value of woody weeds. Front Plant Sci. 2014;5:1-10.

Fountain MT, Hopkin SP. Folsomia candida (Collembola): A standard soil arthropod. Ann Rev Entomol. 2005;50:201-22.

García-Pérez JA, Alarcón-Gutiérrez E, Perroni Y, Barois I. Earthworm communities and soil properties in shaded coffee plantations with and without application of glyphosate. Appl Soil Ecol. 2014;83:230-37. 
Gerlach J, Samways M, Pryke J. Terrestrial invertebrates as bioindicators: an overview of available taxonomic groups. J Insect Conserv. 2013;17:831-50.

Gill HK, Garg H. Pesticides: environmental impacts and management strategies. In: Larramendy ML, Soloneski S, editors. Pesticides - Toxic aspects. Rijeka: InTech; 2014. p.187-230.

Haque A, Gupta R, Chakravorty PP. Effect of two herbicides on Xenylla welchi (Hexapoda: Collembola) under laboratory conditions. Bull Environ Contam Toxicol. 2011;86:583-6.

Hendrix PF, Parmelee RW. Decomposition, nutrient loss and microarthropod densities in herbicide-treated grass litter in a Georgia Piedmont agroecosystem. Soil Biol Biochem. 1985;17:421-8.

Holl KD, Loik ME, Lin EHV, Samuels IA. Tropical montane forest restoration in Costa Rica: overcoming barriers to dispersal and establishment. Restor Ecol. 2000;8:339-49.

Karpouzas DG, Papadopoulou E, Ipsilantis I, Friedel I, Petric I, Udikovic-Kolic N, Djuric S, Kandeler E, Menkissoglu-Spiroudi U, Martin-Laurent F. Effects of nicosulfuron on the abundance and diversity of arbuscular mycorrhizal fungi used as indicators of pesticide soil microbial toxicity. Ecol Indic. 2014;39:44-53.

Machado VM, Santos JB, Pereira IM, Lara RO, Cabral CM, Amaral CS. Sensibilidade de mudas de espécies florestais nativas ao glyphosate. Biosci J. 2013;29:1941-51.

Martinazzo R, Dick DP, Hirsch MM, Leite SB, Peralba MCR. Sorção de atrazina e de mesotriona em Latossolos e estimativa do potencial de contaminação. Quim Nova. 2011;34:1378-84.

Menta C. Soil fauna diversity - function, soil degradation, biological indices, soil restoration. In: Lameed GA, editor. Biodiversity conservation and utilization in a diverse world. Rijeka: InTech; 2012. p.59-94.

Mirsal IA. Soil pollution: origin, monitoring and remediation. Berlin: Springer; 2008.
Mitchell G, Bartlett DW, Fraser TEM, Hawkes TR, Holt DC, Townson JK, Wichert RA. Mesotrione: a new selective herbicide for use in maize. Pest Manage Sci. 2001;57:120-8.

Niva CC, Kovalski R, Brown GG. Adaptação do ensaio ecotoxicológico com enquitreídeos (Enchytraeidae, Oligochaeta). In: Anais do $11^{\circ}$ Congresso Brasileiro de Ecotoxicologia; 2010; Bombinhas. Bombinhas: Universidade do Vale do Itajaí; 2010.

Odum EP, Barrett GW. Fundamentos de ecologia. São Paulo: Cengage Learning; 2011.

Pesaro M, Widmer F, Nicollier G, Zeyer J. Effects of freeze-thaw stress during soil storage on microbial communities and methidathion degradation. Soil Biol Biochem. 2003;35:1049-61.

Rodrigues APC, Castilhos ZC, Cesar RG, Almosny NRP, Linde-Arias AR, Bidone ED. Avaliação de risco ecológico: conceitos básicos, metodologia e estudo de caso. Rio de Janeiro: Cetem/MCT; 2011.

Shepard J, Creighton J, Duzan H. Forestry herbicides in the United States: an overview. Wildlife Soc B. 2004;32:1020-7.

Toledo REB, Alves PLCA, Alves C, Alvarenga SF. Comparação dos custos de quatro métodos de manejo de Brachiaria decumbes Stapf em áreas de Eucalyptus grandis W. Hill ex Maiden. R Árvore. 1996;20:319-30.

United States Environmental Protection Agency - Usepa. Guidelines for Ecological Risk Assessment. Washington, DC: 1998.

Victoria Filho R. Tipos de herbicidas para uso em florestas. Série Técnica IPEF. 1987;4:36-44.

Wang Y, Wu S, Chen L, Wu C, Yu R, Wang Q, Zhao X. Toxicity assessment of 45 pesticides to the epigeic earthworm Eisenia fetida. Chemosphere. 2012;88:484-91.

Zhao J, Neher DA, Fu S, Li Z, Wang K. Non-target effects of herbicides on soil nematode assemblages. Pest Manage Sci. 2013;69:679-84. 